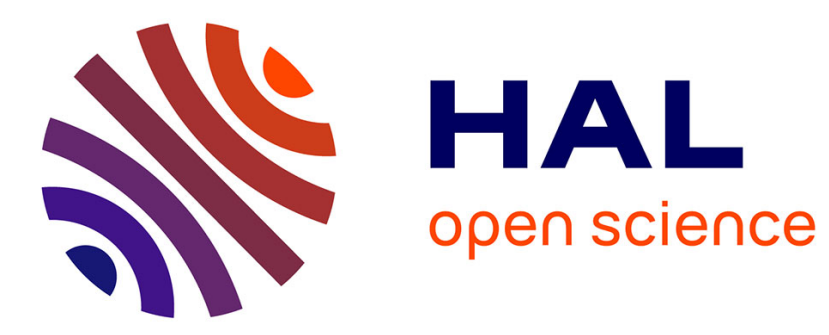

\title{
Structure colomnaire de cristaux liquides
}

D. Guillon, A. Skoulios

\section{To cite this version:}

D. Guillon, A. Skoulios. Structure colomnaire de cristaux liquides. Journal de Physique IV Proceedings, 1996, 06 (C4), pp.C4-41-C4-48. 10.1051/jp4:1996405 . jpa-00254288

\section{HAL Id: jpa-00254288 https://hal.science/jpa-00254288}

Submitted on 1 Jan 1996

HAL is a multi-disciplinary open access archive for the deposit and dissemination of scientific research documents, whether they are published or not. The documents may come from teaching and research institutions in France or abroad, or from public or private research centers.
L'archive ouverte pluridisciplinaire HAL, est destinée au dépôt et à la diffusion de documents scientifiques de niveau recherche, publiés ou non, émanant des établissements d'enseignement et de recherche français ou étrangers, des laboratoires publics ou privés. 


\title{
Structure colomnaire de cristaux liquides
}

\author{
D. Guillon et A. Skoulios
}

Institut de Physique et Chimie des Matériaux de Strasbourg, Groupe des Matériaux Organiques, 23 rue du Loess, 67037 Strasbourg cedex, France

Résumé : Les cristaux liquides sont des systèmes ordonnés caractérisés par des réseaux cristallins à une ou deux dimensions pour les phases smectiques et colomnaires, ou caractérisés par une simple orientation macroscopique des molécules pour la phase nématique. La structure des cristaux liquides colomnaires sera illustrée dans la suite par deux exemples : celui des dérivés de la phthalocyanine, et celui des alkanoates de cuivre (II).

\begin{abstract}
Liquid crystals are ordered systems characterised by one- and two-dimensional crystalline lattices for the smectic and columnar phases, or characterised by only a macroscopic orientation of the molecules for the nematic phase. In the following, will be described two examples of the structure of columnar liquid crystals : the first one deals with phthalocyanine derivatives, the second one with copper (II) alkanoates.
\end{abstract}

\section{INTRODUCTION}

Les cristaux liquides, découverts en 1888 par le biochimiste Reinitzer [1], donnent naissance à des phases intermédiaires entre la phase solide tridimensionnelle et le liquide isotrope ; cet état intermédiaire est aussi appelé état mésomorphe. Les cristaux liquides sont classés en deux catégories principales : les lyotropes dont les mésophases apparaissent par l'addition d'un solvant approprié à un composé organique, et les thermotropes pour lesquels les phases mésomorphes apparaissent par la variation de la seule température. Selon l'architecture des entités moléculaires considérées, on observe soit des phases lamellaires avec des molécules de forme allongée, soit des phases colomnaires avec des molécules en forme de disque par exemple.

Dès 1922, les cristaux liquides obtenus avec des molécules calamitiques ont été classés en deux grandes catégories par G. Friedel [2] : les cristaux liquides nématiques (figure 1a) ( $(\eta \mu \alpha:$ nema, fil) «à cause des discontinuités linéaires contournées comme des fils » observées avec une préparation de ces composés entre lame et lamelle sous microscope en lumière polarisée ; et les cristaux liquides smectiques (figures $1 \mathrm{~b}$ et $1 \mathrm{c})(\sigma \mu \eta \gamma \mu \alpha$ : smegma, savon), car, dans certaines conditions de température et de concentration, les savons appartiennent à cette catégorie. Tandis que le premier groupe possède uniquement un ordre orientationnel, le second groupe possède en plus un ordre positionnel, les molécules se trouvant parallèles entre elles à l'intérieur de couches équidistantes superposées les unes sur 
les autres. Les différents arrangements des centres de gravité des molécules au sein des couches permettent de définir une grande variété de phases smectiques : les phases smectiques dites désordonnées pour lesquelles l'arrangement latéral des molécules est de type liquide, comme les phases smectiques A (orientation normale des molécules par rapport au plan des couches) ou smectiques $C$ (molécules inclinées par rapport au plan des couches) ; d'autres phases smectiques sont dites ordonnées, les centres de gravité des molécules se situant alors aux noeuds d'un réseau périodique bidimensionnel s'étendant à longue portée.

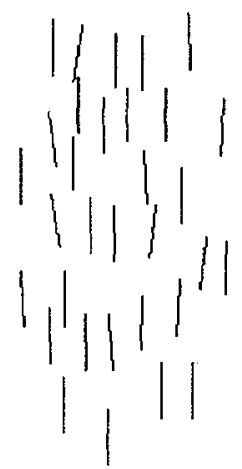

1a : Nématique

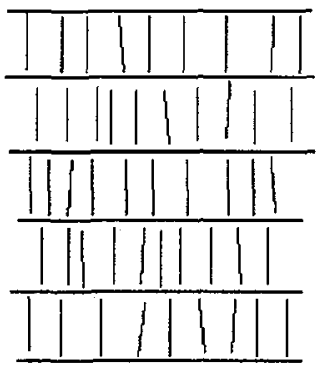

$1 b$ : Smectique $A$

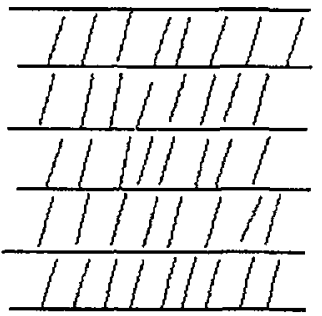

lc: Smectique C

Figure 1 : Représentation schématique des phases nématique et smectiques Schematic representation of nematic and smectic phases

Une grande part de l'intérêt porté ces dernières années aux recherches dans le domaine des mésophases colomnaires a son origine dans la découverte en 1977 par Chandrasekhar [3] de cristaux liquides obtenus à partir de molécules organiques en forme de disque. Ces molécules discotiques s'empilent les unes sur les autres pour former des colonnes de longueur indéfinie, ces colonnes s'organisant selon divers réseaux cristallins bidimensionnels, et le désordre positionnel dans chaque colonne étant plus ou moins prononcé. Les colonnes sont donc en général formées par l'association d'un grand nombre de molécules avec des types variés d'arrangement géométrique en leur sein ; la dimension latérale des colonnes est de l'ordre de quelques nanomètres et est comparable aux dimensions moléculaires.

La diffraction des rayons $\mathrm{X}$ aux petits et aux grands angles de Bragg permet de décrire précisément l'arrangement moléculaire au sein des édifices lamellaires ou colomnaires de ces phases fluides. Nous allons discuter dans la suite la structure colomnaire mésomorphe présentée par deux familles de cristaux liquides thermotropes.

\section{MESOPHASES COLOMNAIRES DE DERIVES DE LA PHTHALOCYANINE}

Les molécules de phthalocyanine avec huit longues chaînes alkyles périphériques présentent des mésophases colomnaires stables thermodynamiquement sur un très grand intervalle de température. En chauffant, les chaînes alkyles deviennent désordonnées au moment de la 
transition de la phase cristalline à la phase liquide-cristalline, et le système adopte une structure correspondant à l'empilement des coeurs aromatiques en colonnes latéralement arrangées selon un réseau hexagonal bidimensionnel [4-9]. Les traits caractéristiques des dérivés de la phthalocyanine, comparés à ceux d'autres molécules discotiques produisant aussi des cristaux liquides colomnaires sont nombreux ; leur coeur aromatique plan est exceptionnellement large et offre ainsi la possibilité d'une délocalisation très large d'électrons $\pi$ et la possibilté de fortes interactions de van der Waals, conduisant ainsi à une remarquable stabilité thermique des mésophases colomnaires ; leur architecture moléculaire offre aussi la possibilité d'inclure un grand nombre d'atomes métalliques dans la cavité centrale du coeur aromatique, ouvrant ainsi la perspective d'obtenir des systèmes métalliques unidimensionnels avec des propriétés conductrices potentielles intéressantes.

L'étude structurale qui suit concerne l'empilement intracolomnaire pour trois séries de dérivés de la phthalocyanine dont la structure chimique est représentée dans la figure 2 . Elle essaie aussi de mettre en relief le mode d'empilement des molécules avec les exigences spécifiques de l'assemblage moléculaire.

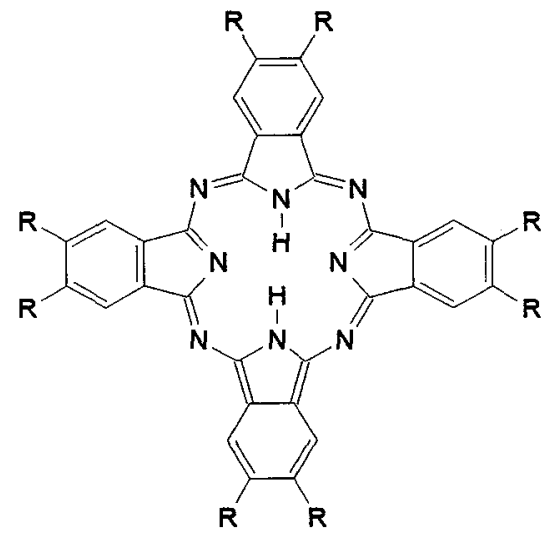

Figure 2 : Structure chimique des molécules de phthalocyanine octasubstituées étudiées : alkoxy : $\mathrm{R}=-\mathrm{O}-\left(\mathrm{CH}_{2}\right)_{n}-\mathrm{CH}_{3} ;$ alkoxyméthyle $: \mathrm{R}=-\mathrm{CH}_{2}-\mathrm{O}-\left(\mathrm{CH}_{2}\right)_{n}-\mathrm{CH}_{3} ;$ alkyle $=-\left(\mathrm{CH}_{2}\right)_{n}-\mathrm{CH}_{3}$. Chemical structure of the octasubstituted phthalocyanine molecules studied: alkoxy : $\mathrm{R}=-\mathrm{O}-\left(\mathrm{CH}_{2}\right)_{n}-\mathrm{CH}_{3} ;$ alkoxymethyl $: \mathrm{R}=-\mathrm{CH}_{2}-\mathrm{O}-\left(\mathrm{CH}_{2}\right)_{n}-\mathrm{CH}_{3} ;$ alkyl $=-\left(\mathrm{CH}_{2}\right)_{n}-\mathrm{CH}_{3}$.

Deux modes d'empilement ont été trouvés. Avec des chaînes périphériques de type alkoxy, les coeurs aromatiques sont orientés perpendiculairement à l'axe colomnaire ; avec les chaînes de type alkyle ou alkoxyméthyle, ils sont inclinés d'un angle de $46^{\circ}$, comme dans la forme cristalline $\beta$ des phthalocyanines non substituées, consolidée par ponts hydrogène. Dans les deux cas, les coeurs aromatiques sont au contact les uns des autres, et superposés d'une manière plutôt régulière. De la sorte, les mésophases colomnaires de ces composés peuvent être classées dans la catégorie des phases colomnaires ordonnées. La différence entre les empilements normaux et inclinés est bien établie expérimentalement et de plus en parfait accord avec les données de la littérature sur les composés aromatiques [10]. Ces données tendent à montrer que, quand ils sont fixés directement au système aromatique planaire, les atomes d'oxygène exercent une influence déterminante sur la structure cristalline ; par l'intermédiaire d'interactions hydrogène faibles, ils induisent des empilements normaux plutôt qu'inclinés. 
En ce qui concerne" l'arrangement latéral des colonnes, qui est réalisé selon un réseau hexagonal bidimensionnel (donnant lieu à des réflexions de Bragg correspondant à des espacements réciproques dans le rapport $1, \sqrt{3}, \sqrt{4}, \sqrt{7} \ldots$ ) trois modes d'arrangement ont été observés selon la forme des coeurs des colonnes et selon leur orientation azimutale par rapport à leur axe [11]. Avec des chaînes périphériques de type alkoxy, les coeurs aromatiques des colonnes ont une forme pratiquement circulaire et leur orientation rotationnelle par rapport à la maille élémentaire est sans influence sur la symétrie du système. En revanche, avec les chaînes de type alkyle ou alkoxyméthyle, les coeurs colomnaires aromatiques ont une forme elliptique, et leur orientation par rapport au réseau hexagonal devient de première importance. Ainsi, pour des chânes courtes (contenant moins que huit atomes de carbone), la section des colonnes est fortement anisotrope ; l'ordre orientationnel azimuthal des colonnes est alors bien développé, et leur arrangement latéral se fait selon une configuration en chevron (Figure 3). Pour des longues chaînes, la section des colonnes est beaucoup moins anisotrope et l'arrangement hexagonal des colonnes est réalisé par suite d'un désordre rotationnel complet autour de l'axe colomnaire. Enfin pour des chaînes de longueur intermédiaire (par exemple contenant douze atomes de carbone), l'assemblage colomnaire se fait dans une configuration en chevrons à basse température et dans une configuration avec désordre rotationnel complet à haute température ; la transition entre les deux modes d'assemblage s'effectue à une température bien précise et rappelle une transition du second ordre.

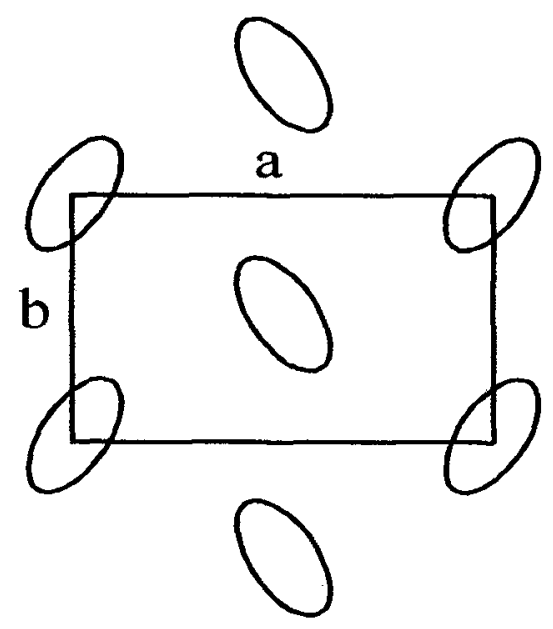

Figure 3 : Représentation schématique de l'arrangement en chevrons des colonnes de section elliptique. Schematic representation of a herringbone arrangement for the elliptical columns.

\section{STRUCTURE COLOMNAIRE DE MOLECULES AMPHIPHILES}

Il existe un intérêt considérable à concevoir des matériaux moléculaires contenant des métaux pour des propriétés physiques spécifiques comme le ferromagnétisme ou leur conductivité électrique par exemple $[12,13]$. Le comportement mésomorphe peut alors être d'une aide précieuse dans l'obtention des propriétés souhaitées, dans la mesure où il est en général facile d'orienter macroscopiquement un cristal liquide, ce qui le rend 
potentiellement utilisable dans certaines applications en électronique. Les complexes de métaux de transition sont à cet égard de bons candidats pour l'élaboration de tels matériaux moléculaires, dans la mesure où ils contiennent des électrons $d$ non-appariés, ce qui peut conduire à l'obtention de certaines propriétés magnétiques ou de transport d'électrons. La connaissance de la structure de ces matériaux dans leur différentes phases est une étape essentielle pour l'analyse de leurs propriétés.

Les alkanoates de cuivre (II) à chaînes aliphatiques de longueur variable (contenant de 5 à 24 atomes de carbone) présentent une structure lamellaire cristalline à température ordinaire, et génèrent une mésophase colomnaire à des températures supérieures à $120^{\circ} \mathrm{C}$ en général [14]. A l'état cristallin, une caractéristique de ces alkanoates est la nature binucléaire de leurs groupes polaires. Dans chaque groupe polaire, qui a globalement la forme d'un prisme quadratique, deux atomes métalliques sont complexés par quatre ligands carboxylates pontants (Figure 4).

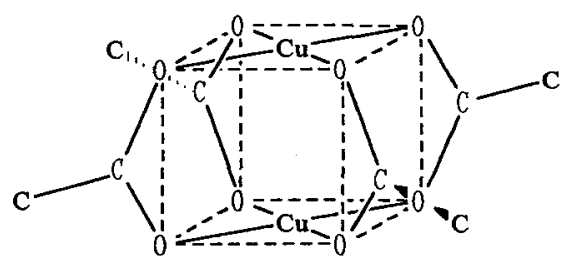

Figure 4 : Structure binucléaire des alkanoates de cuivre (II) Binuclear structure of copper (II) alkanoates

Les atomes métalliques sont complexés axialement avec les atomes d'oxygène des complexes adajacents. Cela conduit à un enchaînement pseudo-polymérique « unidimensionnel » comme montré dans la figure 5.

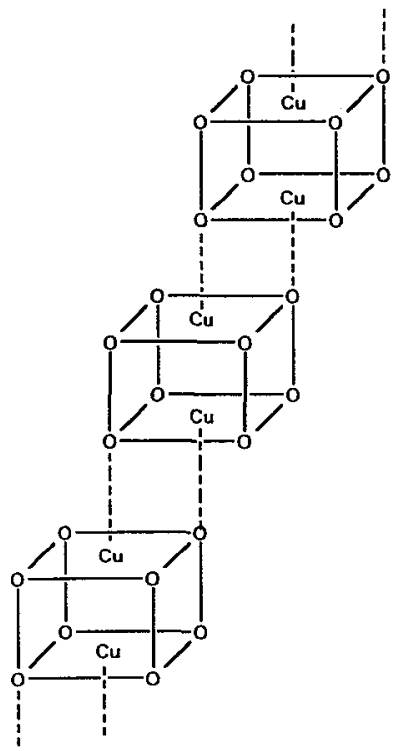

Figure 5 : Enchainement pseudo-polymere des têtes polaires des alkanoates de cuivre (II) Pseudo-polymeric chain of polar heads of copper(II) alkanoates 
L'organisation supramoléculaire correspond alors à l'assemblage de ces colonnes qui, parallèles les unes aux autres, se juxtaposent pour former des lamelles parallèles et équidistantes.

La spectroscopie Exafs au seuil $K$ du cuivre a clairement démontré que les entités binucléaires rencontrées dans la phase cristalline continuent d'exister dans la mésophase colomnaire [15]. En d'autres termes, l'arrangement des têtes polaires correspond au même environnement local des atomes de cuivre dans les deux structures. Par contre, alors que dans la phase cristalline, les chaînes pseudo-polymères de complexes binucléaires résultent de la superposition de ces complexes avec décalage diagonal toujours dans la même direction, dans la phase mésomorphe, elles résultent de la superposition des complexes avec décalage hélicoīdal suivant les quatre positions du complexe plan-carré. La transition entre les deux phases se traduit par une variation discontinue de la période d'empilement suivant l'axe colomnaire, laquelle passe de $5.2 \AA$ pour le cristal à $4.64 \AA$ pour la mésophase.

La structure de la mésophase correspond donc à l'existence de colonnes constituées de l'empilement des têtes polaires de savons arrangées en complexes binucléaires orientés normalement par rapport à l'axe colomnaire, les chaînes aliphatiques désordonnées remplissant l'espace entre les coeurs rigides des colonnes. Orientées parallèlement les unes aux autres, ces colonnes sont arrangées suivant un réseau bidimensionnel hexagonal comme illustré dans la Figure 6.

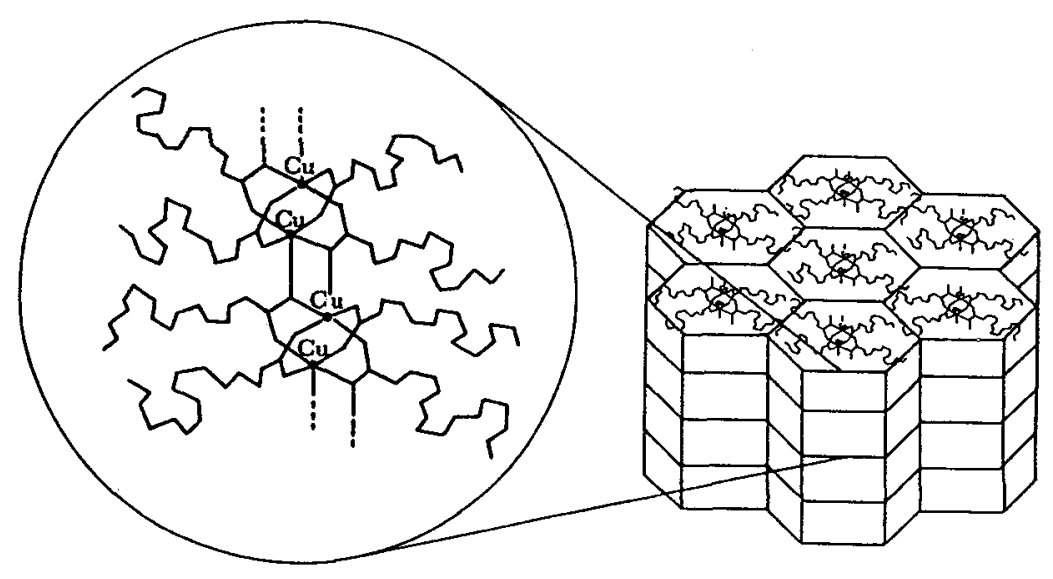

Figure 6: Vue schématique de la mésophase colomnaire des alkanoates de cuivre (II). Schematic view of the columnar mesophase of copper (II) alkanoates.

Une étude par diffraction des rayons $X$ sous pression a montré que l'effet de la pression sur la structure colomnaire est anisotrope. En effet, si l'aire de la maille hexagonale diminue lorsque la pression augmente, la période d'empilement des complexes binucléaires dans les colonnes, elle, augmente de manière inattendue, en tendant vers la valeur observée dans l'état cristallin (Figure 7) [16]. Cette augmentation de la période d'empilement va de pair avec une inclinaison progressive des têtes polaires des molécules. Cette évolution peut s'expliquer très simplement par l'introduction de défauts le long de l'hélice (par omission 
par exemple d'un atome d'oxygène dans la permutation circulaire, ou par changement du pas hélicoïdal).

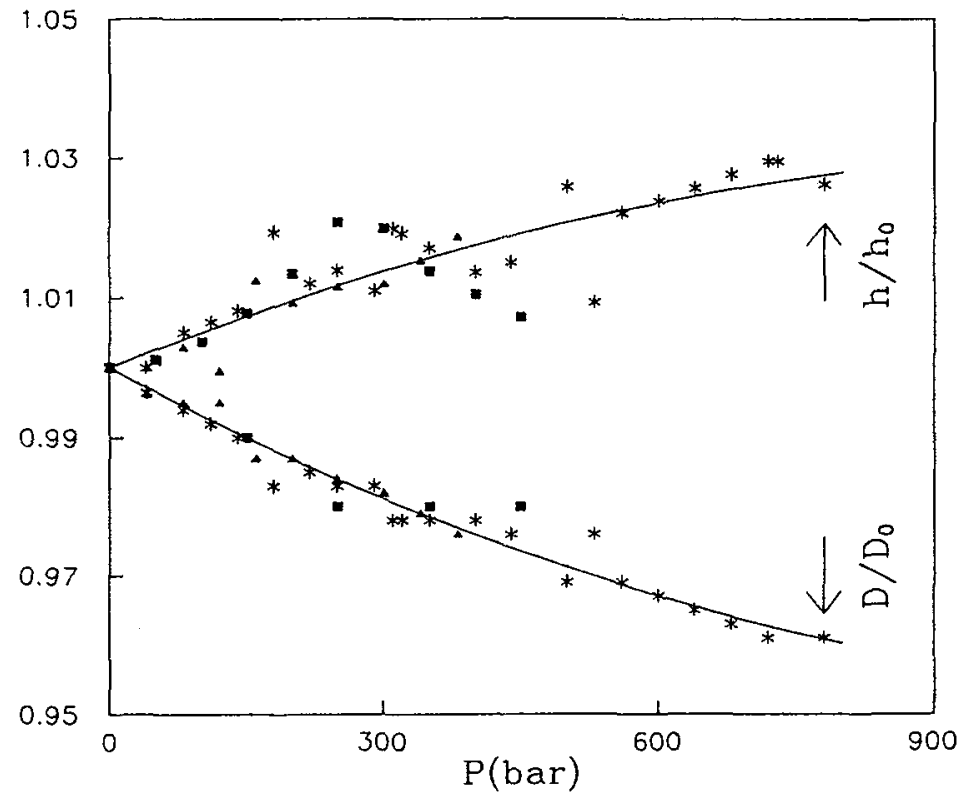

Figure 7 : Variation en fonction de la pression de l'espacement intercolomnaire relatif $D / D_{0}$ et de la période d'empilement intracolomnaire $h / h_{0}$ de divers alkanoates de cuivre (II). $D_{o}$ et $h_{0}$ correspondent a $P=0$. Pressure dependence of the relative intercolumnar spacing $D / D_{o}$ and the relative intracolumnar stacking period $h h_{o}$ of several copper (II) alkanoates. $D_{0}$ and $h_{0}$ correspond to zero pressure.

\section{CONCLUSION}

Les deux exemples discutés ci-dessus illustrent quelques aspects structuraux que l'on peut rencontrer dans les mésophases colomnaires. Le même type d'ordre mésomorphe se rencontre avec d'autres systèmes moléculaires variés, comme des polymères a chaînes latérales dont le squelette est suffisamment rigide pour assurer une ossature solide à chaque colonne [17], comme aussi des molécules polycaténaires constituées d'un coeur rigide allongé prolongé à chaque extrémité de plusieurs chaînes aliphatiques [18], ou comme enfin des systèmes à transfert de charge [19]. Mais si Georges Friedel avait su déduire la structure des phases mésomorphes lamiellaires de certains composés, grâce à de simples observations au microscope et à un raisonnement de géométrie, les techniques de diffraction et d'absorption des rayons $\mathrm{X}$ se sont révélées depuis indispensables pour décrire l'ordre à la fois intra- et intermoléculaire au sein des phases liquide-cristallines. 


\section{Remerciements.}

Les auteurs remercient A.M. Giroud, P. Maldivi, J.C. Marchon (CENG, Grenoble) et J. Simon (ESPCI) pour la synthèse des composés étudiés, et pour leur collaboration fructueuse dans l'ananlyse des études structurales.

\section{Références}

[1] Reinitzer, F., Monatsh. 9 (1888) 421.

[2] Friedel M.G., Ann. de Phys. 18 (1922) 19.

[3] Chandrasekhar S., Sadashiva K., Suresh K.A., Pramana 9 (1977) 471.

[4] Piechocki C., Simon J., Skoulios A., Guillon D., Weber P., J. Am. Chem. Soc. 104 (1982) 5245.

[5] Guillon D., Weber P., Skoulios A., Piechocki C., Simon J., Mol. Cryst. Liq. Cryst. 130 (1985) 223.

[6] Hanack M., Beck A., Lehmann H., Synthesis Commun. 8 (1987) 703.

[7] Sauer T., Wegner G., Mol. Cryst. Liq. Cryst. 162 (1988) 97.

[8] van der Pol J.F., Neeleman E., Zwikker J.W., Nolte R.J.M., Drenth W. Recl. Trav. Chim. Pays Bas Belg. 107 (1988) 615 ; van der Pol J.F., Neeleman E., Zwikker J.W., Nolte R.J.M., Drenth, Aerts J., Picken S.J., Liq. Cryst. 6 (1989) 577.

[9] M.J. Cook, J. Mat. Sci. Mater. Electron. 5 (1994) 117.

[10] Dsiraju D.R., Radhan Kishan, J. Am. Chem. Soc. 111 1989) 4838.

[11] Weber, P., Guillon, D., Skoulios, A., Liq. Cryst. 9 (1991) 369.

[12] Kahn O., Molecular Magnetism (VCH Publishers, 1993).

[13] Göpel W., Ziegler Ch., Nanostructures Based on Molecular Materials (VCH Publishers, 1992).

[14] Ibn-Elhaj, M., Guillon, D., Skoulios, Giroud-Godquin, A.M., Marchon, J.C., Liq. Cryst. 11 (1992) 731.

[15] Abied, H., Guillon, D., Skoulios, A., Dexpert, H., Giroud-Godquin, A.M., Marchon, J.C., J. de Physique 49 (1988) 345.

[16] Ibn-Elhaj, M., Guillon, D., Skoulios, A., Phys. Rev. A 46 (1992) 7643 ;

[17] Weber P., Guillon D., Skoulios A., Miller R.D., Liq. Cryst. 8 (1990) 825.

[18] Malthête J., Levelut A.M., Nguyen H.T., J. Phys. Lett. (Paris) 46 (1985) L875 ; Guillon D., Skoulios A., Malthête J., J. Phys. (Paris) 3 (1987) 67 ; Nguyen H.T., Destrade C., Levelut A.M., Malthête J., J. Phys. (Paris) 47 (1986) 553 ; Destrade C., Nguyen H.T., Roubineau A., Levelut A.M., Mol. Cryst. Liq. Cryst. 159 (1988) 163.

[19] Singer D., Liebmann A., Praefcke K., Wendorff J.H., Liq. Cryst. 14 (1993) 785; Praefcke K., Bilgin B., Pickardt J., Borowski M., Chem. Ber. 127 (1994) 1543. 